\title{
Avaliação do estado nutricional da soja
}

\author{
Eliane Oshiro Mocelin Urano(1), Carlos Hissao Kurihara(2), Shizuo Maeda ${ }^{(2)}$, Antonio Carlos Tadeu Vitorino ${ }^{(3)}$, \\ Manoel Carlos Gonçalves ${ }^{(3)}$ e Marlene Estevão Marchetti(3)
}

\begin{abstract}
(1)Sementes Barreirão Ltda., Rod. BR 376, Km 09, Caixa Postal 1005, CEP 79830-970 Dourados, MS. E-mail: elimocelin@terra.com.br (2)Embrapa Agropecuária Oeste, Rod. BR 163, Km 253, Caixa Postal 661, CEP 79804-970 Dourados, MS. E-mail: kurihara@cpao.embrapa.br, maeda@cpao.embrapa.br (3)Universidade Federal de Mato Grosso do Sul, Dep. de Ciências Agrárias, Caixa Postal 533, CEP $74804-970$ Dourados, MS. E-mail: vitorino@ceud.ufms.br, mancgonc@ceud.ufms.br, emarche@ceud.ufms.br
\end{abstract}

Resumo - O objetivo deste trabalho foi comparar os diagnósticos do estado nutricional de um conjunto de 111 lavouras comerciais de soja, pelos métodos: Sistema Integrado de Diagnose e Recomendação (DRIS) e Diagnose da Composição Nutricional (CND), com uso do conceito de potencial de resposta à adubação. Nas lavouras analisadas, observou-se que Zn, P e Fe foram os nutrientes diagnosticados por ambos os métodos como os mais limitantes pela ausência, e Mg e Mn os mais limitantes por excesso. A freqüência de lavouras com diagnósticos concordantes, quanto ao potencial de resposta à adubação, foi maior para os nutrientes considerados de maior deficiência relativa (90,3\%), em relação aos considerados de maior excesso relativo (81,9\%). Quando se avaliaram os diagnósticos concordantes quanto ao estado nutricional, a freqüência variou de 85,9\% para o P a 98,6\% para o Mn. Os métodos DRIS e CND tendem a ser concordantes quanto ao diagnóstico nutricional; o agrupamento de classes de potencial de resposta à adubação pode resultar no agrupamento, em uma única classe de estado nutricional, os nutrientes com alta e baixa probabilidade de resposta à adubação.

Termos para indexação: Glycine max, diagnose foliar, deficiência nutricional.

\section{Soybean nutritional status evaluation}

\begin{abstract}
The objetive of of this work was to compare the diagnostics of the nutritional state of a group of 111 commercial soybean plantations, obtained by Diagnosis and Recommendation Integrated System (DRIS) and Compositional Nutrient Diagnosis (CND) methods applying the response potencial to fertilization concept. It was observed that zinc, phosphorus and iron were the nutrients diagnosed by both methods as the more restrictive for lack, and magnesium and manganese the more restrictive for excess. Agreement between DRIS and CND diagnoses was higher for nutrients considered of greater relative deficiency (90.3\%) than for those considered of greater relative excess (81.9\%). When the classes of nutritional state were evaluated, the frequency of plantations with concordant diagnosis varied from $85.9 \%$ for phosphorus to $98.6 \%$ for manganese. DRIS and CND methods tend to be consonants about nutritional status diagnoses; classes grouping of potential response to fertilization may result in grouping in one class of nutritional state nutrients with high and low probability of response.
\end{abstract}

Index terms: Glycine max, foliar diagnosis, nutritional deficiency.

\section{Introdução}

A avaliação do estado nutricional de espécies vegetais, baseada na interpretação de resultados de análise foliar, tem sido amplamente utilizada e discutida, no intuito de possibilitar intervenções mais precisas em sistemas de produção vegetal, por meio de práticas de manejo e adubação.

Diversos estudos têm demonstrado a natureza dinâmica da composição de nutrientes no tecido vegetal, em conseqüência de fatores como idade da planta (Beverly, 1993), condições climáticas (Walworth \& Sumner, 1987) e de solo (Kurihara, 2004). Além disso, variações no teor de um nutriente podem influenciar não somente o teor de outro nutriente no tecido vegetal, mas também o valor crítico deste (Bailey et al., 1997).Assim, a necessidade de uma combinação específica de teores de nutrientes, para a obtenção de produtividade elevada, tem sido considerada em métodos como o Sistema Integrado de Diagnose e Recomendação (DRIS) (Wadt, 1996; Wadt et al., 1999; Silva, 2001; Reis Junior \& Monnerat, 2003; Kurihara, 2004) e Diagnose da Composição Nutricional (CND) (Parent \& Dafir, 1992; Silva, 2001; Khiari et al., 2001a, 2001b; Kurihara, 2004). 
Enquanto o emprego do método DRIS pressupõe que as relações entre os teores de dois nutrientes (relações bivariáveis) são as melhores indicadoras do desequilíbrio nutricional (Wadt, 1996), as relações entre o teor de um nutriente e a média geométrica dos teores dos demais componentes da matéria seca, inclusive aqueles não avaliados analiticamente (relações multivariáveis), são consideradas como forma de expressão do equilíbrio nutricional, quando se emprega o método CND (Parent \& Dafir, 1992).

A compreensão dos princípios considerados por diferentes métodos de diagnose, bem como a comparação de seus resultados, é importante para a utilização criteriosa destas ferramentas de análise.

Objetivo deste trabalho foi comparar os diagnósticos do estado nutricional de um conjunto de 111 lavouras comerciais de soja, na região sul do Estado de Mato Grosso do Sul, realizados pelos métodos DRIS e CND.

\section{Material e Métodos}

Foram feitas amostragens foliares em 27 lavouras comerciais de soja, cultivadas no sistema plantio direto, no ano agrícola 2001/2002, na região sul do Estado de Mato Grosso do Sul. Em cada lavoura, coletaram-se amostras do terceiro trifólio com pecíolo, do ápice para a base na haste principal, em 30 plantas, no estádio de desenvolvimento R2 (Costa \& Marquezan, 1982), que constituíram uma amostra composta. No estádio R8 (Costa \& Marquezan, 1982), a produtividade de grãos foi determinada a partir da amostragem de três linhas de cultivo, com $2 \mathrm{~m}$ de comprimento cada, em quatro locais escolhidos aleatoriamente na área demarcada.

Constituíram o banco de dados, além das 27 amostras já mencionadas, 84 amostras coletadas por Maeda (2002) no ano agrícola 2000/2001, no estádio de desenvolvimento R1, em lavouras comerciais cultivadas no sistema plantio direto, na região sul do Estado de Mato Grosso do Sul. As análises químicas de ambos os conjuntos de amostras de tecido foliar seguiram os procedimentos indicados por Malavolta et al. (1997). Desse conjunto de amostras do banco de dados foi definida como subpopulação de alta produtividade, aquelas amostras cujo rendimento de grãos foi superior à média mais 0,5 desvio-padrão (4.399 $\mathrm{kg} \mathrm{ha}^{-1}$ ).
A hipótese de normalidade da distribuição de freqüências foi aceita para 58,2\% dos quocientes, entre teores de nutrientes, na subpopulação de alta produtividade. Por sua vez, a transformação logarítmica neperiana possibilitou a aceitação da hipótese testada em 94,5\% dos quocientes na subpopulação de alta produtividade, razão pela qual, após esta transformação, a média aritmética e o desvio-padrão de todos os quocientes das relações diretas e inversas, conforme sugerido por Alvarez Venegas \& Leite (1999), entre os teores de nutrientes na população de alta produtividade, constituíram as normas ou valores-padrão, em função dos quais se procedeu à avaliação do estado nutricional pelo método DRIS (Tabela 1).

Depois da transformação, por função logarítmica neperiana, dos quocientes entre teores de nutrientes em uma amostra (A/B, A/C, A/N, ..., B/A, C/A, N/A), os desvios destes $[\mathrm{Z}(\mathrm{A} / \mathrm{B}), \mathrm{Z}(\mathrm{A} / \mathrm{C}), \mathrm{Z}(\mathrm{A} / \mathrm{N}), \ldots, \mathrm{Z}(\mathrm{B} / \mathrm{A})$, $\mathrm{Z}(\mathrm{C} / \mathrm{A}), \mathrm{Z}(\mathrm{N} / \mathrm{A})]$, em relação aos valores médios dos mesmos quocientes, na população de alta produtividade $(\mathrm{a} / \mathrm{b}, \mathrm{a} / \mathrm{c}, \mathrm{a} / \mathrm{n}, \ldots, \mathrm{b} / \mathrm{a}, \mathrm{c} / \mathrm{a}, \mathrm{n} / \mathrm{a})$, foram então determinados de acordo com Jones (1981), em unidades de desvio-padrão (s), utilizando-se um fator de ajuste $(\mathrm{c})=1$, conforme sugerido por Wadt et al. (1998): Z(A/B) $=[(\mathrm{A} / \mathrm{B})-(\mathrm{a} / \mathrm{b})](\mathrm{c} / \mathrm{s})$.

Os índices DRIS para cada nutriente consistiram da média aritmética das relações diretas (A/B) e inversas (B/A), transformadas em variáveis normais reduzidas, de acordo com Alvarez Venegas \& Leite (1999), em que $n$ é o número de nutrientes envolvidos na análise: $\mathrm{I}_{\mathrm{A}}=[\mathrm{Z}(\mathrm{A} / \mathrm{B})+\mathrm{Z}(\mathrm{A} / \mathrm{C})+\mathrm{Z}(\mathrm{A} / \mathrm{N})+\ldots-\mathrm{Z}(\mathrm{B} / \mathrm{A})-\mathrm{Z}(\mathrm{C} / \mathrm{A})-$ $\mathrm{Z}(\mathrm{N} / \mathrm{A})] / 2(\mathrm{n}-1)$.

As variáveis multinutrientes $\left(\mathrm{V}_{\mathrm{A}}\right)$ consistiram do logaritmo neperiano do quociente entre a concentração de cada nutriente (A) e a média geométrica das concentrações dos constituintes da massa de matéria seca (G), adaptado de Khiari et al. (2001a, 2001b): $\mathrm{V}_{\mathrm{A}}=\ln (\mathrm{A} / \mathrm{G})$.

A aceitação da hipótese de distribuição normal das freqüências ocorreu em 90,9\% das variáveis multinutrientes na subpopulação de referência (Tabela 2), sendo as normas CND constituídas dos valores médios e desvios-padrão destas, conforme Khiari et al. (2001a, 2001b).

Os índices CND foram calculados pela diferença entre as variáveis multinutriente, no talhão avaliado $\left(\mathrm{V}_{\mathrm{A}}\right)$ e na média da população de referência $\left(\mathrm{V}_{\mathrm{A}}{ }^{*}\right)$, dividido pelo desvio-padrão desta variável, na população de referência $\left(\mathrm{S}_{\mathrm{A}} *\right)$ : $\mathrm{I}_{\mathrm{A}}=\left(\mathrm{V}_{\mathrm{A}}-\mathrm{V}_{\mathrm{A}}{ }^{*}\right) / \mathrm{S}_{\mathrm{A}} *$. 
Tabela 1. Médias (y) e desvio-padrão (s) dos quocientes entre teores ( $\mathrm{mg} \mathrm{kg}^{-1}$ ) de nutrientes em folhas de soja, na subpopulação de alta produtividade ${ }^{(1)}$, transformadas por função logarítmica neperiana, em amostras coletadas na região sul do Estado de Mato Grosso do Sul, nos anos agrícolas de 2000/2001 e 2001/2002.

\begin{tabular}{|c|c|c|c|c|c|c|c|}
\hline Variável & $\bar{y}$ & $\mathrm{~s}$ & $\mathrm{p}^{(2)}$ & Variável & $\overline{\mathrm{y}}$ & $\mathrm{s}$ & $\mathrm{p}^{(2)}$ \\
\hline $\mathrm{N} / \mathrm{P}$ & 2,6127 & 0,1951 & $>0,15$ & $\mathrm{~S} / \mathrm{B}$ & 3,9783 & 0,3646 & $>0,15$ \\
\hline $\mathrm{N} / \mathrm{K}$ & 0,5651 & 0,2634 & $>0,15$ & $\mathrm{~S} / \mathrm{Cu}$ & 5,5397 & 0,3742 & 0,118 \\
\hline $\mathrm{N} / \mathrm{Ca}$ & 1,2654 & 0,2830 & $>0,15$ & $\mathrm{~S} / \mathrm{Fe}$ & 3,2541 & 0,4039 & 0,013 \\
\hline $\mathrm{N} / \mathrm{Mg}$ & 2,5768 & 0,4004 & 0,053 & $\mathrm{~S} / \mathrm{Mn}$ & 3,7273 & 0,3596 & $>0,15$ \\
\hline $\mathrm{N} / \mathrm{S}$ & 2,8842 & 0,3209 & $>0,15$ & $\mathrm{~S} / \mathrm{Zn}$ & 3,7397 & 0,3705 & $>0,15$ \\
\hline N/B & 6,8625 & 0,1628 & $>0,15$ & $\mathrm{~B} / \mathrm{N}$ & $-6,8625$ & 0,1628 & $>0,15$ \\
\hline $\mathrm{N} / \mathrm{Cu}$ & 8,4239 & 0,4212 & $>0,15$ & $\mathrm{~B} / \mathrm{P}$ & $-4,2497$ & 0,1931 & $>0,15$ \\
\hline $\mathrm{N} / \mathrm{Fe}$ & 6,1383 & 0,3207 & $>0,15$ & $\mathrm{~B} / \mathrm{K}$ & $-6,2974$ & 0,2798 & 0,036 \\
\hline N/Mn & 6,6115 & 0,4152 & $>0,15$ & $\mathrm{~B} / \mathrm{Ca}$ & $-5,5971$ & 0,3242 & $>0,15$ \\
\hline $\mathrm{N} / \mathrm{Zn}$ & 6,6239 & 0,4387 & $>0,15$ & $\mathrm{~B} / \mathrm{Mg}$ & $-4,2856$ & 0,4435 & 0,108 \\
\hline $\mathrm{P} / \mathrm{N}$ & $-2,6127$ & 0,1951 & $>0,15$ & $\mathrm{~B} / \mathrm{S}$ & $-3,9783$ & 0,3646 & $>0,15$ \\
\hline $\mathrm{P} / \mathrm{K}$ & $-2,0476$ & 0,2033 & 0,053 & $\mathrm{~B} / \mathrm{Cu}$ & 1,5615 & 0,4256 & $>0,15$ \\
\hline $\mathrm{P} / \mathrm{Ca}$ & $-1,3473$ & 0,2402 & $>0,15$ & $\mathrm{~B} / \mathrm{Fe}$ & $-0,7241$ & 0,3553 & 0,131 \\
\hline $\mathrm{P} / \mathrm{Mg}$ & $-0,0359$ & 0,3508 & 0,126 & $\mathrm{~B} / \mathrm{Mn}$ & $-0,2509$ & 0,4241 & $>0,15$ \\
\hline $\mathrm{P} / \mathrm{S}$ & 0,2715 & 0,3054 & $>0,15$ & $\mathrm{~B} / \mathrm{Zn}$ & $-0,2386$ & 0,4487 & $>0,15$ \\
\hline $\mathrm{P} / \mathrm{B}$ & 4,2497 & 0,1931 & $>0,15$ & $\mathrm{Cu} / \mathrm{N}$ & $-8,4239$ & 0,4212 & $>0,15$ \\
\hline $\mathrm{P} / \mathrm{Cu}$ & 5,8112 & 0,4001 & 0,062 & $\mathrm{Cu} / \mathrm{P}$ & $-5,8112$ & 0,4001 & 0,062 \\
\hline $\mathrm{P} / \mathrm{Fe}$ & 3,5256 & 0,3034 & $>0,15$ & $\mathrm{Cu} / \mathrm{K}$ & $-7,8589$ & 0,4091 & $>0,15$ \\
\hline $\mathrm{P} / \mathrm{Mn}$ & 3,9988 & 0,3478 & $>0,15$ & $\mathrm{Cu} / \mathrm{Ca}$ & $-7,1586$ & 0,3966 & $>0,15$ \\
\hline $\mathrm{P} / \mathrm{Zn}$ & 4,0112 & 0,3604 & $>0,15$ & $\mathrm{Cu} / \mathrm{Mg}$ & $-5,8471$ & 0,4213 & 0,073 \\
\hline $\mathrm{K} / \mathrm{N}$ & $-0,5651$ & 0,2634 & $>0,15$ & $\mathrm{Cu} / \mathrm{S}$ & $-5,5397$ & 0,3742 & 0,118 \\
\hline $\mathrm{K} / \mathrm{P}$ & 2,0476 & 0,2033 & 0,055 & $\mathrm{Cu} / \mathrm{B}$ & $-1,5615$ & 0,4256 & $>0,15$ \\
\hline $\mathrm{K} / \mathrm{Ca}$ & 0,7003 & 0,2498 & 0,066 & $\mathrm{Cu} / \mathrm{Fe}$ & $-2,2856$ & 0,5187 & 0,035 \\
\hline $\mathrm{K} / \mathrm{Mg}$ & 2,0117 & 0,3444 & $>0,15$ & $\mathrm{Cu} / \mathrm{Mn}$ & $-1,8124$ & 0,4159 & $>0,15$ \\
\hline $\mathrm{K} / \mathrm{S}$ & 2,3191 & 0,3075 & $>0,15$ & $\mathrm{Cu} / \mathrm{Zn}$ & $-1,8001$ & 0,4347 & $>0,15$ \\
\hline $\mathrm{K} / \mathrm{B}$ & 6,2974 & 0,2798 & 0,036 & $\mathrm{Fe} / \mathrm{N}$ & $-6,1383$ & 0,3207 & $>0,15$ \\
\hline $\mathrm{K} / \mathrm{Cu}$ & 7,8589 & 0,4091 & $>0,15$ & $\mathrm{Fe} / \mathrm{P}$ & $-3,5256$ & 0,3034 & $>0,15$ \\
\hline $\mathrm{K} / \mathrm{Fe}$ & 5,5733 & 0,3557 & $>0,15$ & $\mathrm{Fe} / \mathrm{K}$ & $-5,5733$ & 0,3557 & $>0,15$ \\
\hline $\mathrm{K} / \mathrm{Mn}$ & 6,0465 & 0,3559 & $>0,15$ & $\mathrm{Fe} / \mathrm{Ca}$ & $-4,8730$ & 0,2930 & $>0,15$ \\
\hline $\mathrm{K} / \mathrm{Zn}$ & 6,0588 & 0,3693 & $>0,15$ & $\mathrm{Fe} / \mathrm{Mg}$ & $-3,5615$ & 0,3917 & $>0,15$ \\
\hline $\mathrm{Ca} / \mathrm{N}$ & $-1,2654$ & 0,2830 & $>0,15$ & $\mathrm{Fe} / \mathrm{S}$ & $-3,2541$ & 0,4039 & 0,013 \\
\hline $\mathrm{Ca} / \mathrm{P}$ & 1,3473 & 0,2402 & $>0,15$ & $\mathrm{Fe} / \mathrm{B}$ & 0,7241 & 0,3553 & 0,131 \\
\hline $\mathrm{Ca} / \mathrm{K}$ & $-0,7003$ & 0,2498 & 0,066 & $\mathrm{Fe} / \mathrm{Cu}$ & 2,2856 & 0,5187 & 0,035 \\
\hline $\mathrm{Ca} / \mathrm{Mg}$ & 1,3114 & 0,2003 & $>0,15$ & $\mathrm{Fe} / \mathrm{Mn}$ & 0,4732 & 0,4316 & $>0,15$ \\
\hline $\mathrm{Ca} / \mathrm{S}$ & 1,6188 & 0,3051 & $>0,15$ & $\mathrm{Fe} / \mathrm{Zn}$ & 0,4855 & 0,4275 & $>0,15$ \\
\hline $\mathrm{Ca} / \mathrm{B}$ & 5,5971 & 0,3242 & $>0,15$ & $\mathrm{Mn} / \mathrm{N}$ & $-6,6115$ & 0,4152 & $>0,15$ \\
\hline $\mathrm{Ca} / \mathrm{Cu}$ & 7,1586 & 0,3966 & $>0,15$ & $\mathrm{Mn} / \mathrm{P}$ & $-3,9988$ & 0,3478 & $>0,15$ \\
\hline $\mathrm{Ca} / \mathrm{Fe}$ & 4,8730 & 0,2930 & $>0,15$ & $\mathrm{Mn} / \mathrm{K}$ & $-6,0465$ & 0,3559 & $>0,15$ \\
\hline $\mathrm{Ca} / \mathrm{Mn}$ & 5,3462 & 0,3059 & $>0,15$ & $\mathrm{Mn} / \mathrm{Ca}$ & $-5,3462$ & 0,3059 & $>0,15$ \\
\hline $\mathrm{Ca} / \mathrm{Zn}$ & 5,3585 & 0,3036 & $>0,15$ & $\mathrm{Mn} / \mathrm{Mg}$ & $-4,0347$ & 0,3433 & 0,063 \\
\hline $\mathrm{Mg} / \mathrm{N}$ & $-2,5768$ & 0,4004 & 0,053 & $\mathrm{Mn} / \mathrm{S}$ & $-3,7273$ & 0,3596 & $>0,15$ \\
\hline $\mathrm{Mg} / \mathrm{P}$ & 0,0359 & 0,3508 & 0,126 & $\mathrm{Mn} / \mathrm{B}$ & 0,2509 & 0,4241 & $>0,15$ \\
\hline $\mathrm{Mg} / \mathrm{K}$ & $-2,0117$ & 0,3444 & $>0,15$ & $\mathrm{Mn} / \mathrm{Cu}$ & 1,8124 & 0,4159 & $>0,15$ \\
\hline $\mathrm{Mg} / \mathrm{Ca}$ & $-1,3114$ & 0,2003 & $>0,15$ & $\mathrm{Mn} / \mathrm{Fe}$ & $-0,4732$ & 0,4316 & $>0,15$ \\
\hline $\mathrm{Mg} / \mathrm{S}$ & 0,3074 & 0,3060 & $>0,15$ & $\mathrm{Mn} / \mathrm{Zn}$ & 0,0123 & 0,3220 & $>0,15$ \\
\hline $\mathrm{Mg} / \mathrm{B}$ & 4,2856 & 0,4435 & 0,108 & $\mathrm{Zn} / \mathrm{N}$ & $-6,6239$ & 0,4387 & $>0,15$ \\
\hline $\mathrm{Mg} / \mathrm{Cu}$ & 5,8471 & 0,4213 & 0,073 & $\mathrm{Zn} / \mathrm{P}$ & $-4,0112$ & 0,3604 & $>0,15$ \\
\hline $\mathrm{Mg} / \mathrm{Fe}$ & 3,5615 & 0,3917 & $>0,15$ & $\mathrm{Zn} / \mathrm{K}$ & $-6,0588$ & 0,3693 & $>0,15$ \\
\hline $\mathrm{Mg} / \mathrm{Mn}$ & 4,0347 & 0,3433 & 0,063 & $\mathrm{Zn} / \mathrm{Ca}$ & $-5,3585$ & 0,3036 & $>0,15$ \\
\hline $\mathrm{Mg} / \mathrm{Zn}$ & 4,0470 & 0,3371 & $>0,15$ & $\mathrm{Zn} / \mathrm{Mg}$ & $-4,0470$ & 0,3371 & $>0,15$ \\
\hline $\mathrm{S} / \mathrm{N}$ & $-2,8842$ & 0,3209 & $>0,15$ & $\mathrm{Zn} / \mathrm{S}$ & $-3,7397$ & 0,3705 & $>0,15$ \\
\hline $\mathrm{S} / \mathrm{P}$ & $-0,2715$ & 0,3054 & $>0,15$ & $\mathrm{Zn} / \mathrm{B}$ & 0,2386 & 0,4487 & $>0,15$ \\
\hline $\mathrm{S} / \mathrm{K}$ & $-2,3191$ & 0,3075 & $>0,15$ & $\mathrm{Zn} / \mathrm{Cu}$ & 1,8001 & 0,4347 & $>0,15$ \\
\hline $\mathrm{S} / \mathrm{Ca}$ & $-1,6188$ & 0,3051 & $>0,15$ & $\mathrm{Zn} / \mathrm{Fe}$ & $-0,4855$ & 0,4275 & $>0,15$ \\
\hline $\mathrm{S} / \mathrm{Mg}$ & $-0,3074$ & 0,3060 & $>0,15$ & $\mathrm{Zn} / \mathrm{Mn}$ & $-0,0123$ & 0,3220 & $>0,15$ \\
\hline
\end{tabular}

${ }^{(1)}$ Maior que a média $+0,5$ s, com $n=39$ talhões. ${ }^{(2)}$ Probabilidade para aceitação da hipótese de normalidade de distribuição de freqüências, pelo teste de Kolmogorov-Smirnov (Campos, 1983). 
O somatório dos valores em módulo dos índices DRIS ou CND, de todos os nutrientes, constituíram o índice de equilíbrio nutricional (IEN) da amostra sob diagnose: IEN $=\left|I_{A}\right|+\left|I_{B}\right|+\left|I_{C}\right|+\ldots+\left|I_{N}\right|$.

O índice de equilíbrio nutricional médio (IENm) foi obtido (Wadt, 1996), dividindo-se o valor do índice de equilíbrio nutricional (IEN) pelo número de nutrientes avaliados (n): IENm = IEN/n.

A interpretação dos índices DRIS e CND foi realizada tendo-se classificado os nutrientes de acordo com o potencial de resposta à adubação (PRA) (Wadt, 1996).

Para maior síntese, as classes de potencial de resposta positiva (p) e positiva ou nula (pz) e, também, negativa (n) e negativa ou nula (nz), foram agrupadas e denominadas de limitantes por falta (LF) ou por excesso (LE). E a classe de resposta nula (z) foi denominada como não limitante (NL), conforme Silva (2001).

Verificou-se, para os métodos DRIS e CND, a hipótese de que a freqüência com que cada nutriente ocorreu como o limitante primário por deficiência, ou seja, com potencial de resposta à adubação positiva e de alta probabilidade, tenha sido atribuída ao acaso. Para isto, utilizou-se o teste de qui-quadrado de ajustamento (Silva, 2001), a 5\% de probabilidade, com $\mathrm{n}-1$ graus de liberdade $(\mathrm{n}=$ número de nutrientes analisados).

Tabela 2. Média (y) e desvio-padrão (s) das variáveis multinutrientes e média geométrica dos constituintes da massa seca $(G)$, em folhas de soja coletadas na região sul do Estado de Mato Grosso do Sul, nos anos agrícolas de 2000/2001 e 2001/2002, na subpopulação de alta produtividade ${ }^{(1)}$.

\begin{tabular}{lrrc}
\hline Variável & \multicolumn{1}{c}{$\overline{\mathrm{y}}$} & \multicolumn{1}{c}{$\mathrm{p}^{(2)}$} \\
\hline $\mathrm{G}$ & $1.277,380$ & 142,600 & \multicolumn{1}{c}{${ }^{n}$} \\
$\mathrm{~V}_{\mathrm{N}}{ }^{*}$ & 3,453 & 0,205 & $>0,150$ \\
$\mathrm{~V}_{\mathrm{P}}{ }^{*}$ & 0,840 & 0,151 & $>0,150$ \\
$\mathrm{~V}_{\mathrm{K}}{ }^{*}$ & 2,888 & 0,186 & 0,0874 \\
$\mathrm{~V}_{\mathrm{Ca}}{ }^{*}$ & 2,188 & 0,152 & $>0,150$ \\
$\mathrm{~V}_{\mathrm{Mg}}{ }^{*}$ & 0,876 & 0,250 & 0,1333 \\
$\mathrm{~V}_{\mathrm{S}}{ }^{*}$ & 0,569 & 0,223 & $>0,150$ \\
$\mathrm{~V}_{\mathrm{B}}{ }^{*}$ & $-3,409$ & 0,234 & 0,0937 \\
$\mathrm{~V}_{\mathrm{Cu}}{ }^{*}$ & $-4,971$ & 0,328 & 0,0945 \\
$\mathrm{~V}_{\mathrm{Fe}}{ }^{*}$ & $-2,685$ & 0,279 & 0,0169 \\
$\mathrm{~V}_{\mathrm{Mn}}{ }^{*}$ & $-3,159$ & 0,271 & $>0,150$ \\
$\mathrm{~V}_{\mathrm{Zn}}{ }^{*}$ & $-3,171$ & 0,284 & $>0,150$ \\
\hline
\end{tabular}

(1)Maior que a média $+0,5 \mathrm{~s}$, com $\mathrm{n}=39$ talhões. (2)Probabilidade para aceitação da hipótese de normalidade de distribuição de frequêencias,

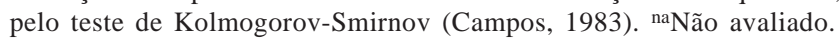

Considerou-se como o limitante primário por deficiência o nutriente que apresentou o índice negativo, de menor valor em relação aos índices dos demais nutrientes, e maior em módulo que o índice de equilíbrio nutricional médio (IENm). Se verdadeira a hipótese considerada, as freqüências observadas para todos os nutrientes seriam estatisticamente iguais entre si.

As freqüências esperadas (FE) e observadas (FO) foram calculadas da seguinte forma: FE $(\%)=\left[\left(n^{0}\right.\right.$ total de talhões avaliados $/ n^{0}$ de nutrientes avaliados $) / n^{0}$ total de talhões avaliados] x 100; e FO (\%) = (no de talhões em que o nutriente foi considerado como o limitante primário por deficiência/no total de talhões avaliados) x 100.

\section{Resultados e Discussão}

Entre os 111 talhões amostrados, 39 (35,1\%) apresentaram produtividade superior a $4.399 \mathrm{~kg} \mathrm{ha}^{-1} \mathrm{e}$ constituíram a subpopulação de alta produtividade. Os 72 talhões restantes $(64,9 \%)$ constituíram, portanto, a subpopulação de baixa produtividade. Os valores correspondentes aos teores de nutrientes e produtividade média dos talhões, nas subpopulações de alta e baixa produtividade, estão descritos na Tabela 3.

Tanto pela diagnose realizada pelo método DRIS, quanto na realizada pelo CND, rejeitou-se a hipótese de que as freqüências dos diagnósticos, observadas para todos os nutrientes, eram estatisticamente iguais entre si, o que indica que os métodos foram sensíveis para diagnosticar diferenças de probabilidade de resposta positiva à adubação para os nutrientes avaliados (Tabela 4).

Verificou-se que o nutriente diagnosticado como o de maior probabilidade de resposta negativa, pelo DRIS e CND, foi o Mg, seguido pelo Mn (Tabela 5). É possível que a detecção de excessos de $\mathrm{Mg}$ e $\mathrm{Mn}$, na nutrição da soja, se deva ao uso freqüente de termofosfato magnesiano em lavouras da região amostrada. Esta fonte de $\mathrm{P}$ apresenta em sua composição $17 \%$ de $\mathrm{P}_{2} \mathrm{O}_{5}$ total, $7 \%$ de $\mathrm{Mg}$ e $0,4 \%$ de $\mathrm{Mn}$.

Os nutrientes diagnosticados como de maior probabilidade de resposta positiva à adubação, pelos métodos DRIS e CND, foram o P, Fe e K (Tabela 5). A ocorrência do diagnóstico de potencial de resposta positiva à adubação para o nutriente $\mathrm{Fe}$, determinado pelos métodos DRIS e CND, embora não esperada, ocorreu em talhões em que os teores de Fe, no tecido 
foliar, variaram entre 30,9 e 60,2 $\mathrm{mg} \mathrm{kg}^{-1}$, enquanto o teor ótimo estimado para esse nutriente em soja, em amostras coletadas na região sul do Estado de Mato Grosso do Sul (Urano, 2004) foi de $84 \mathrm{mg} \mathrm{kg}^{-1}$, pelo método da Chance Matemática e 90,1 $\mathrm{mg} \mathrm{kg}^{-1}$ pelos métodos DRIS e CND. De forma semelhante, os teores ótimos de Fe em amostras de soja, coletadas nos estados de Mato Grosso, Mato Grosso do Sul e Goiás, estimados por Kurihara (2004), foram $77 \mathrm{mg} \mathrm{kg}^{-1}$, pelo método da Chance Matemática, e $86 \mathrm{mg} \mathrm{kg}^{-1}$, pelos métodos DRIS e CND.
Ao se avaliar a freqüência de diagnoses concordantes, quanto ao potencial de resposta à adubação, tendo-se considerado cada nutriente separadamente, foi verificado que a menor concordância entre os métodos avaliados ocorreu para o nutriente P $(74,6 \%)$, uma vez que o método CND diagnosticou maior desequilíbrio por deficiência relativa para esse nutriente, em relação ao método DRIS. Para os outros nutrientes avaliados, a freqüência de diagnoses concordantes do potencial de resposta à adubação variou de $85,9 \%$, no caso do $\mathrm{Cu}$, a $94,4 \%$ para o N (Tabela 6).

Tabela 3. Valores mínimos, máximos, médios e desvios-padrão (s) para teores de nutrientes em folhas de soja, e produtividade, em amostras coletadas na região sul do Estado de Mato Grosso do Sul, nos anos agrícolas de 2000/2001 e 2001/2002, nas subpopulações de alta e baixa produtividade ${ }^{(1)}$.

\begin{tabular}{|c|c|c|c|c|c|c|c|c|}
\hline \multirow[t]{2}{*}{ Nutriente } & \multicolumn{4}{|c|}{ Alta produtividade } & \multicolumn{4}{|c|}{ Baixa produtividade } \\
\hline & Mínimo & Máximo & Média & (s) & Mínimo & Máximo & Média & (s) \\
\hline $\mathrm{N}\left(\mathrm{g} \mathrm{kg}^{-1}\right)$ & 29,1 & 56,7 & 40,6 & 6,4 & 27,0 & 53,9 & 39,0 & 6,7 \\
\hline$P\left(\mathrm{~g} \mathrm{~kg}^{-1}\right)$ & 2,2 & 3,7 & 3,0 & 0,4 & 1,3 & 4,2 & 2,7 & 0,6 \\
\hline $\mathrm{K}\left(\mathrm{g} \mathrm{kg}^{-1}\right)$ & 13,0 & 29,0 & 23,1 & 3,8 & 12,0 & 34,5 & 22,1 & 4,5 \\
\hline $\mathrm{Ca}\left(\mathrm{g} \mathrm{kg}^{-1}\right)$ & 7,8 & 18,0 & 11,6 & 2,7 & 4,4 & 17,8 & 10,7 & 2,3 \\
\hline $\operatorname{Mg}\left(\mathrm{g} \mathrm{kg}^{-1}\right)$ & 1,8 & 5,0 & 3,2 & 1,0 & 1,7 & 6,0 & 3,6 & 1,0 \\
\hline $\mathrm{S}\left(\mathrm{g} \mathrm{kg}^{-1}\right)$ & 1,3 & 4,3 & 2,3 & 0,6 & 1,4 & 3,5 & 2,4 & 0,4 \\
\hline $\mathrm{B}\left(\mathrm{mg} \mathrm{kg}^{-1}\right)$ & 26,9 & 61,0 & 42,7 & 7,9 & 23,8 & 59,7 & 42,6 & 7,3 \\
\hline $\mathrm{Cu}\left(\mathrm{mg} \mathrm{kg}^{-1}\right)$ & 2,6 & 17,2 & 9,4 & 3,3 & 4,2 & 15,3 & 9,6 & 3,3 \\
\hline $\mathrm{Fe}\left(\mathrm{mg} \mathrm{kg}^{-1}\right)$ & 32,0 & 164,9 & 90,1 & 24,8 & 30,9 & 240,8 & 78,9 & 31,2 \\
\hline $\operatorname{Mn}\left(\mathrm{mg} \mathrm{kg}^{-1}\right)$ & 19,3 & 122,9 & 57,2 & 20,2 & 19,0 & 170,0 & 68,4 & 30,7 \\
\hline $\mathrm{Zn}\left(\mathrm{mg} \mathrm{kg}^{-1}\right)$ & 29,0 & 122,0 & 56,8 & 20,9 & 21,0 & 174,0 & 49,9 & 24,0 \\
\hline Produtividade $\left(\mathrm{kg} \mathrm{ha}^{-1}\right)$ & 4.410 & 5.583 & 4.701 & 290 & 2.363 & 4.393 & 3.750 & 499 \\
\hline
\end{tabular}

(1)Maior e menor que a média + 0,5 desvio-padrão, com número de talhões de 39 e 72 para as subpopulações de alta e baixa produtividade, respectivamente.

Tabela 4. Cálculo do qui-quadrado, para as freqüências observadas (\%) de N, P, K, Ca, Mg, S, B, Cu, Fe, Mn e Zn como nutriente limitante primário por deficiência(1), pelo método DRIS e CND, na suposição de que as freqüências observadas são conseqüência do acaso, em amostras de soja coletadas na região sul do Estado de Mato Grosso do Sul, na subpopulação de baixa produtividade $^{(2)}$.

\begin{tabular}{|c|c|c|c|c|c|c|}
\hline \multirow[t]{2}{*}{ Nutriente } & \multicolumn{3}{|c|}{ DRIS } & \multicolumn{3}{|c|}{ CND } \\
\hline & $\mathrm{FO}^{(3)}$ & $\mathrm{FE}^{(3)}$ & $(\mathrm{FO}-\mathrm{FE}) / \mathrm{FE}$ & $\mathrm{FO}^{(3)}$ & $\mathrm{FE}^{(3)}$ & $(\mathrm{FO}-\mathrm{FE}) / \mathrm{FE}$ \\
\hline $\bar{N}$ & 4,17 & 9,09 & 2,67 & 4,17 & 9,09 & 2,67 \\
\hline $\mathrm{P}$ & 12,50 & 9,09 & 1,28 & 18,06 & 9,09 & 8,84 \\
\hline K & 13,89 & 9,09 & 2,53 & 13,89 & 9,09 & 2,53 \\
\hline $\mathrm{Ca}$ & 9,72 & 9,09 & 0,04 & 12,50 & 9,09 & 1,28 \\
\hline $\mathrm{Mg}$ & 5,56 & 9,09 & 1,37 & 5,56 & 9,09 & 1,37 \\
\hline S & 1,39 & 9,09 & 6,53 & 1,39 & 9,09 & 6,53 \\
\hline B & 5,56 & 9,09 & 1,37 & 5,56 & 9,09 & 1,37 \\
\hline $\mathrm{Cu}$ & 12,50 & 9,09 & 1,28 & 11,11 & 9,09 & 0,45 \\
\hline $\mathrm{Fe}$ & 16,67 & 9,09 & 6,31 & 12,50 & 9,09 & 1,28 \\
\hline $\mathrm{Mn}$ & 6,94 & 9,09 & 0,51 & 5,56 & 9,09 & 1,37 \\
\hline $\mathrm{Zn}$ & 11,11 & 9,09 & 0,45 & 9,72 & 9,09 & 0,04 \\
\hline Qui-quadr & & & $24,34 * *$ & & & $27,74 * * *$ \\
\hline
\end{tabular}

${ }^{(1)}$ Com potencial de resposta à adubação positiva e muito provável (p), conforme Wadt (1996). (2)Menor que a média + 0,5 s. (3)FO e FE correspondem às freqüências observada e esperada, respectivamente. ** e ***p menor que 0,02 e 0,01 , respectivamente. 
Wadt (1996) sugere que a recomendação de adubação seja realizada para o nutriente que apresente alta probabilidade de resposta positiva. De modo análogo, também é interessante a adoção de práticas de manejo que possibilitem, mesmo que a longo prazo, a redução do suprimento relativo do nutriente com alta probabilidade de resposta negativa à adubação. Por essa razão, avaliaram-se as freqüências de diagnoses concordantes, entre os dois métodos, quanto ao potencial de resposta à adubação, para as classes de alta probabilidade de resposta positiva (p) e alta probabilidade de resposta negativa (n).
Observaram-se elevados níveis de concordância para essas duas classes de probabilidade de resposta à adubação, tendo sido maior a concordância para os nutrientes considerados de maior deficiência relativa (90,3\%), do que para os nutrientes de maior excesso relativo $(81,9 \%)$ (Tabela 7$)$. Resultados semelhantes foram obtidos por Silva (2001), ao determinar a freqüência de talhões com diagnoses concordantes, para as classes de resposta positiva de alta probabilidade (88,8\%) e negativa de alta probabilidade (80,4\%), em talhões florestais de eucalipto, pelos métodos DRIS e CND.

Tabela 5. Freqüência (\%) do potencial de resposta à adubação de N, P, K, Ca, Mg, S, B, Cu, Fe, Mn e Zn, determinada pelos métodos DRIS e CND, em amostras de soja coletadas na região sul do Estado de Mato Grosso do Sul, nos anos agrícolas de 2000/2001 e 2001/2002, na subpopulação de baixa produtividade ${ }^{(1)}$.

\begin{tabular}{|c|c|c|c|c|c|c|}
\hline \multirow[t]{2}{*}{ Nutriente } & \multirow[t]{2}{*}{ Método } & \multicolumn{5}{|c|}{ Potencial de resposta à adubação $0^{(2)}$} \\
\hline & & $p$ & $\mathrm{pz}$ & $\mathrm{Z}$ & $\mathrm{nz}$ & $\mathrm{n}$ \\
\hline \multirow[t]{2}{*}{$\mathrm{N}$} & DRIS & 4,2 & 13,9 & 68,1 & 12,5 & 1,4 \\
\hline & CND & 4,2 & 15,3 & 65,3 & 11,1 & 4,2 \\
\hline \multirow[t]{2}{*}{$\mathrm{P}$} & DRIS & 12,5 & 18,1 & 61,1 & 6,9 & 1,4 \\
\hline & CND & 18,1 & 20,8 & 47,2 & 4,2 & 9,7 \\
\hline \multirow[t]{2}{*}{ K } & DRIS & 13,9 & 5,6 & 69,4 & 9,7 & 1,4 \\
\hline & CND & 13,9 & 8,3 & 68,1 & 6,9 & 2,8 \\
\hline \multirow[t]{2}{*}{$\mathrm{Ca}$} & DRIS & 9,7 & 8,3 & 77,8 & 2,8 & 1,4 \\
\hline & CND & 12,5 & 12,5 & 68,1 & 4,2 & 2,8 \\
\hline \multirow[t]{2}{*}{$\mathrm{Mg}$} & DRIS & 5,6 & 13,9 & 34,7 & 13,9 & 31,9 \\
\hline & CND & 5,6 & 12,5 & 36,1 & 16,7 & 29,2 \\
\hline \multirow[t]{2}{*}{ S } & DRIS & 1,4 & 4,2 & 72,2 & 18,1 & 4,2 \\
\hline & CND & 1,4 & 4,2 & 72,2 & 13,9 & 8,3 \\
\hline \multirow[t]{2}{*}{ B } & DRIS & 5,6 & 2,8 & 75,0 & 13,9 & 2,8 \\
\hline & CND & 5,6 & 2,8 & 80,6 & 9,7 & 1,4 \\
\hline \multirow[t]{2}{*}{$\mathrm{Cu}$} & DRIS & 12,5 & 6,9 & 48,6 & 18,1 & 13,9 \\
\hline & CND & 11,1 & 8,3 & 54,2 & 16,7 & 9,7 \\
\hline \multirow[t]{2}{*}{$\mathrm{Fe}$} & DRIS & 16,7 & 16,7 & 51,4 & 8,3 & 6,9 \\
\hline & CND & 12,5 & 18,1 & 55,6 & 9,7 & 4,2 \\
\hline \multirow[t]{2}{*}{$\mathrm{Mn}$} & DRIS & 6,9 & 8,3 & 41,7 & 13,9 & 29,2 \\
\hline & CND & 5,6 & 9,7 & 43,1 & 19,4 & 22,2 \\
\hline \multirow[t]{2}{*}{$\mathrm{Zn}$} & DRIS & 11,1 & 27,8 & 50,0 & 5,6 & 5,6 \\
\hline & CND & 9,7 & 25,0 & 55,6 & 4,2 & 5,6 \\
\hline
\end{tabular}

${ }^{(1)}$ Menor que a média $+0,5$ s. ${ }^{(2)}$ p: positiva, com alta probabilidade; pz: positiva, com baixa probabilidade; z: nula; nz: negativa, com baixa probabilidade; n: negativa, com alta probabilidade, conforme Wadt (1996).

Tabela 6. Freqüência (\%) de talhões com diagnoses concordantes do potencial de resposta à adubação (PRA) e do estado nutricional (EN), determinados pelos métodos DRIS e CND, em amostras de soja coletadas na região sul do Estado de Mato Grosso do Sul, nos anos agrícolas de 2000/2001 e 2001/2002, na subpopulação de baixa produtividade ${ }^{(1)}$.

\begin{tabular}{lccccccccccc}
\hline & $\mathrm{N}$ & $\mathrm{P}$ & $\mathrm{K}$ & $\mathrm{Ca}$ & $\mathrm{Mg}$ & $\mathrm{S}$ & $\mathrm{B}$ & $\mathrm{Cu}$ & $\mathrm{Fe}$ & $\mathrm{Mn}$ & $\mathrm{Zn}$ \\
\hline PRA $^{(2)}$ & 94,4 & 74,6 & 91,5 & 85,9 & 93,0 & 93,0 & 90,1 & 85,9 & 88,7 & 90,1 & 93,0 \\
$\mathrm{EN}^{(3)}$ & 97,2 & 85,9 & 93,0 & 90,1 & 95,8 & 97,2 & 91,5 & 94,4 & 95,8 & 98,6 & 94,4 \\
\hline
\end{tabular}

${ }^{(1)}$ Menor que a média $+0,5 \mathrm{~s}$. 
De maneira semelhante, Parent et al. (1994), ao avaliar desequilíbrios nutricionais em plantios de batata, observaram alta correlação entre os índices DRIS e CND; e Raghupathi \& Bhargava (1998) verificaram alta correlação entre os índices de equilíbrio nutricional, produzidos para diagnósticos de romãzeiros, pelos métodos DRIS e CND, que indicaram, também, boa concordância no diagnóstico do desequilíbrio geral de nutrientes por ambos os métodos.

Agrupando-se as classes de potencial de resposta à adubação positiva (p) e positiva ou nula (pz), em uma classe de estado nutricional denominada limitante por falta (LF); as classes de potencial de resposta à adubação negativa (n) e negativa ou nula (nz), em uma classe denominada limitante por excesso (LE); e as de resposta nula (z), em uma classe denominada não limitante (NL), conforme Silva (2001), pôde-se verificar maior proporção de talhões (45,8\%), em que o Mg foi diagnosticado como o nutriente mais limitante por excesso, seguido do Mn (43,1 e 41,7\%, pelo DRIS e CND, respectivamente). Zn, $\mathrm{P}$ e Fe foram diagnosticados, com maior freqüência, como os nutrientes mais limitantes por falta, pelos métodos DRIS e CND (Tabela 8).

Deve-se observar, entretanto, que a indicação do Zn como o nutriente para o qual houve maior freqüência de limitação por falta, por ambos os métodos (38,9 e 34,7\%, para o DRIS e CND, respectivamente) (Tabela 8), deveu-se à considerável proporção de talhões em que o nutriente foi diagnosticado como de baixa probabilidade de resposta positiva (Tabela 5).

Isto indica que embora o agrupamento de classes de potencial de resposta à adubação em classes de estado nutricional, conforme sugerido por Silva (2001), permita maior síntese dos resultados, esse procedimento pode

Tabela 7. Freqüência de talhões com diagnoses concordantes do potencial de resposta à adubação (p e $n)^{(1)}$, entre os métodos DRIS e CND, em amostras de soja coletadas na região sul do Estado de Mato Grosso do Sul, nos anos agrícolas de 2000/2001 e 2001/2002, na subpopulação de baixa produtividade ${ }^{(2)}$.

\begin{tabular}{lcc}
\hline Métodos & $\mathrm{p}$ & $\mathrm{n}$ \\
\hline DRIS vs. CND & 90,3 & 81,9 \\
\hline
\end{tabular}

${ }^{(1)} \mathrm{p}$ = positiva, com alta probabilidade; n: negativa, com alta probabilidade, conforme Wadt (1996). ${ }^{(2)}$ Menor que a média + 0,5 s. resultar no agrupamento em uma única classe de estado nutricional de nutrientes com alta e baixa probabilidade de resposta à adubação.

Quando se avaliou a freqüência de diagnoses concordantes quanto ao estado nutricional, considerando-se os nutrientes isoladamente, a menor concordância foi verificada para P (85,9\%) e a maior para o Mn (98,6\%) (Tabela 6). Esse resultado já era esperado, uma vez que ao se adotar apenas três classes de estado nutricional, ao invés de cinco classes de potencial de resposta à adubação, a probabilidade de existir concordância é maior.

Verifica-se, portanto, que a depender da forma de comparação escolhida, a avaliação dos nutrientes quanto ao potencial de resposta à adubação ou quanto à classe de estado nutricional, pode ter resultados diferentes quanto à concordância entre os métodos DRIS e CND.

Tabela 8. Freqüência (\%) de diagnose do estado nutricional de N, P, K, Ca, Mg, S, B, Cu, Fe, Mn e Zn, determinado pelos métodos DRIS e CND, em amostras de soja coletadas na região sul do Estado de Mato Grosso do Sul, nos anos agrícolas de 2000/2001 e 2001/2002, na subpopulação de baixa produtividade $^{(1)}$.

\begin{tabular}{lcrcr}
\hline Nutriente & Método & \multicolumn{3}{c}{ Estado nutricional $^{(2)}$} \\
\cline { 3 - 5 } & & LF & NL & LE \\
\hline $\mathrm{N}$ & DRIS & 18,1 & 68,1 & 13,9 \\
$\mathrm{P}$ & CND & 19,4 & 65,3 & 15,3 \\
& DRIS & 30,6 & 61,1 & 8,3 \\
$\mathrm{~K}$ & CND & 38,9 & 47,2 & 13,9 \\
& DRIS & 19,4 & 69,4 & 11,1 \\
$\mathrm{Ca}$ & CND & 22,2 & 68,1 & 9,7 \\
& DRIS & 18,1 & 77,8 & 4,2 \\
$\mathrm{Mg}$ & CND & 25,0 & 68,1 & 6,9 \\
& DRIS & 19,4 & 34,7 & 45,8 \\
$\mathrm{~S}$ & CND & 18,1 & 36,1 & 45,8 \\
& DRIS & 5,6 & 72,2 & 22,2 \\
$\mathrm{~B}$ & CND & 5,6 & 72,2 & 22,2 \\
& DRIS & 8,3 & 75,0 & 16,7 \\
$\mathrm{Cu}$ & CND & 8,3 & 80,6 & 11,1 \\
& DRIS & 19,4 & 48,6 & 31,9 \\
$\mathrm{Fe}$ & CND & 19,4 & 54,2 & 26,4 \\
& DRIS & 33,3 & 51,4 & 15,3 \\
$\mathrm{Mn}$ & CND & 30,6 & 55,6 & 13,9 \\
& DRIS & 15,3 & 41,7 & 43,1 \\
$\mathrm{Zn}$ & CND & 15,3 & 43,1 & 41,7 \\
& DRIS & 38,9 & 50,0 & 11,1 \\
& CND & 34,7 & 55,6 & 9,7 \\
\hline
\end{tabular}

${ }^{(1)}$ Menor que a média + 0,5 s. (2)LF: limitante por falta; NL: não limitante; LE: limitante por excesso, conforme Silva (2001). 


\section{Conclusões}

1) Os métodos DRIS e CND tendem a ser concordantes quanto ao diagnóstico nutricional.

2) O agrupamento de classes de potencial de resposta à adubação pode resultar no agrupamento em uma única classe de estado nutricional, de nutrientes com alta e baixa probabilidade de resposta à adubação.

\section{Referências}

ALVAREZ VENEGAS, V.H.; LEITE, R. de A. Fundamentos estatísticos das fórmulas usadas para cálculo dos índices DRIS. Boletim Informativo da Sociedade Brasileira de Ciência do Solo, v.24, p.20-25, 1999.

BAILEY, J.S.; BEATTIE, J.A.M.; KILPATRICK, D.J. The diagnosis and recommendation integrated system (DRIS) for diagnosing the nutrient status of grassland swards. I. Model establishment. Plant and Soil, v.197, p.127-135, 1997.

BEVERLY, R.B. Dris diagnoses of soybean nitrogen, phosphorus, and potassium status are unsatisfactory. Journal of Plant Nutrition, v.16, p.1431-1447, 1993.

CAMPOS, H. Estatística experimental não-paramétrica. Piracicaba: USP, 1983. 349p.

COSTA, J.A.; MARQUEZAN, E. Características dos estádios de desenvolvimento da soja. Campinas: Fundação Cargill, 1982. 30p.

JONES, C.A. Proposed modifications of the diagnosis and recommendation integrated system (DRIS) for interpreting plant analyses. Communications in Soil Science and Plant Analysis, v.12, p.785-794, 1981.

KHIARI, L.; PARENT, L.E.; TREMBLAY, N. Critical compositional nutrient indexes for sweet corn at early growth stage. Agronomy Journal, v.93, p.809-814, 2001a.

KHIARI, L.; PARENT, L.E.; TREMBLAY, N. The phosphorus compositional nutrient diagnosis range for potato. Agronomy Journal, v.93, p.815-819, 2001b.

KURIHARA, C.H. Demanda de nutrientes pela soja e diagnose de seu estado nutricional. 2004. 101p. Tese (Doutorado) Universidade Federal de Viçosa, Viçosa.

MAEDA, S. Interpretação do estado nutricional de soja pelo DRIS no Mato Grosso do Sul. 2002. 107p. Dissertação (Doutorado) - Universidade Federal do Paraná, Curitiba.

MALAVOLTA, E.; VITTI, G.C.; OLIVEIRA, S.A. de. Princípios, métodos e técnicas de avaliação do estado nutricional. In:
MALAVOLTA, E.; VITTI, G.C.; OLIVEIRA, S.A. de. Avaliação do estado nutricional de plantas: princípios e aplicações. 2.ed. Piracicaba: Potafos, 1997. p.115-230.

PARENT, L.E.; CAMBOURIS, A.N.; MUHAWENIMANA, A. Multivariate diagnosis of nutrient imbalance in potato crops. Soil Science Society of America Journal, v.58, p.1432-1438, 1994.

PARENT, L.E.; DAFIR, M. A theoretical concept of compositional nutrient diagnosis. Journal of the American Society for Horticultural Science, v.117, p.239-242, 1992.

RAGHUPATHI, H.B.; BHARGAVA, B.S. Diagnosis of nutrient imbalance in pomegranate by diagnosis and recommendation integrated system and compositional nutrient diagnosis. Communications in Soil Science and Plant Analysis, v.29, p.2881-2892, 1998.

REIS JUNIOR, R. dos A.; MONNERAT, P.H. DRIS norms validation for sugarcane crop. Pesquisa Agropecuária Brasileira, v.38, p.379-385, 2003.

SILVA, G.G.C. Diagnose nutricional do eucalipto pelo DRIS, M-DRIS e CWADT, P.G.S.; NOVAIS, R.F. de; ALVAREZ VENEGAS, V.H.; BARROS, N.F. de; DIAS, L.E. Variações no estado nutricional de eucaliptos por influência do material genético e da idade da árvore. Pesquisa Agropecuária Brasileira, v.34, p.17971803, 1999.ces in soil science. New York: Springer-Verlag, 1987. v.6, p.149-188.

URANO, E.O.M. Avaliação do estado nutricional e teores ótimos de nutrientes em soja. 2004. 83p. Dissertação (Mestrado) Universidade Federal de Mato Grosso do Sul, Dourados.

WADT, P.G.S. Os métodos da chance matemática e do sistema integrado de diagnose e recomendação (DRIS) na avaliação nutricional de plantios de eucalipto. 1996. 123p. Tese (Doutorado) - Universidade Federal de Viçosa, Viçosa.

WADT, P.G.S.; NOVAIS, R.F. de; ALVAREZ VENEGAS, V.H.; BARROS, N.F. de; DIAS, L.E. Variações no estado nutricional de eucaliptos por influência do material genético e da idade da árvore. Pesquisa Agropecuária Brasileira, v.34, p.1797-1803, 1999.

WADT, P.G.S.; NOVAIS, R.F. de; ALVAREZ VENEGAS, V.H.; FONSECA, S.; BARROS, N.F. de; DIAS, L.E. Três métodos de cálculo do DRIS para avaliar o potencial de resposta à adubação de árvores de eucalipto. Revista Brasileira de Ciência do Solo, v.22, p.661-666, 1998.

WALWORTH, J.L.; SUMNER, M.E. The diagnosis and recommendation integrated system (DRIS). In: STEWART, B.A. (Ed.). Advances in soil science. New York: Springer-Verlag, 1987. v.6, p.149-188. 